\title{
Characterization of the Abydos region through OSIRIS high-resolution images in support of CIVA measurements
}

\author{
A. Lucchetti ${ }^{1,2}$, G. Cremonese ${ }^{2}$, L. Jorda ${ }^{3}$, F. Poulet ${ }^{4}$, J.-P. Bibring ${ }^{4}$, M. Pajola ${ }^{1}$, F. La Forgia ${ }^{5}$, M. Massironi ${ }^{6}$, \\ M. R. El-Maarry ${ }^{7}$, N. Oklay ${ }^{8}$, H. Sierks ${ }^{8}$, C. Barbieri ${ }^{5}$, P. Lamy ${ }^{3}$, R. Rodrigo ${ }^{9,10}$, D. Koschny ${ }^{11}$, H. Rickman ${ }^{12,13}$, \\ H. U. Keller ${ }^{14}$, J. Agarwal ${ }^{7}$, M. F. A'Hearn ${ }^{15}$, M. A. Barucci ${ }^{16}$, J.-L. Bertaux ${ }^{17}$, I. Bertini ${ }^{1}$, V. Da Deppo ${ }^{18}$, \\ B. Davidsson ${ }^{12}$, S. Debei ${ }^{19}$, M. De Cecco ${ }^{20}$, S. Fornasier ${ }^{16}$, M. Fulle ${ }^{21}$, O. Groussin ${ }^{3}$, P. J. Gutierrez ${ }^{22}$, C. Güttler ${ }^{8}$, \\ S. F. Hviid ${ }^{23}$, W.-H. Ip ${ }^{24}$, J. Knollenberg ${ }^{23}$, J.-R. Kramm ${ }^{8}$, E. Kührt ${ }^{23}$, M. Küppers ${ }^{25}$, L. M. Lara ${ }^{22}$, M. Lazzarin ${ }^{5}$, \\ J. J. Lopez Moreno ${ }^{22}$, F. Marzari ${ }^{5}$, S. Mottola ${ }^{23}$, G. Naletto ${ }^{26,1,17}$, F. Preusker ${ }^{23}$, F. Scholten ${ }^{23}$, N. Thomas ${ }^{7}$, \\ C. Tubiana ${ }^{8}$, and J.-B. Vincent ${ }^{8}$ \\ (Affiliations can be found after the references)
}

Received 8 September 2015 / Accepted 13 November 2015

\begin{abstract}
Context. On 12 November 2014, the European mission Rosetta delivered the Philae lander on the nucleus of comet 67P/ChuryumovGerasimenko (67P). After the first touchdown, the lander bounced three times before finally landing at a site named Abydos.

Aims. We provide a morphologically detailed analysis of the Abydos landing site to support Philae's measurements and to give context for the interpretation of the images coming from the Comet Infrared and Visible Analyser (CIVA) camera system onboard the lander.

Methods. We used images acquired by the OSIRIS Narrow Angle Camera (NAC) on 6 December 2014 to perform the analysis of the Abydos landing site, which provided the geomorphological map, the gravitational slope map, the size-frequency distribution of the boulders. We also computed the albedo and spectral reddening maps.

Results. The morphological analysis of the region could suggest that Philae is located on a primordial terrain. The Abydos site is surrounded by two layered and fractured outcrops and presents a $0.02 \mathrm{~km}^{2}$ talus deposit rich in boulders. The boulder size frequency distribution gives a cumulative power-law index of $-4.0+0.3 /-0.4$, which is correlated with gravitational events triggered by sublimation and/or thermal fracturing causing regressive erosion. The average value of the albedo is $5.8 \%$ at $\lambda_{1}=480.7 \mathrm{~nm}$ and $7.4 \%$ at $\lambda_{2}=649.2 \mathrm{~nm}$, which is similar to the global albedos derived by OSIRIS and CIVA, respectively.
\end{abstract}

Key words. comets: general - comets: individual: 67P/Churyumov-Gerasimenko - methods: data analysis

\section{Introduction}

On 6 August 2014, the ESA Rosetta spacecraft was inserted into orbit around the Jupiter-family comet $67 \mathrm{P} /$ ChuryumovGerasimenko (67P). It delivered the Philae lander on the nucleus on 12 November 2014, when the comet was at 3.0 AU from the Sun. Philae first touched down at 15:34 UT at the selected Agilkia "J" landing site on the head of the nucleus of 67P, where it failed to anchor at the surface. Then, it bounced three times for an additional two-hour flight (Biele et al. 2015) before finally landing at a site later named Abydos, about $1 \mathrm{~km}$ away from the first touchdown. During the lander descent the comparison between high-resolution images acquired by the ROsetta Lander Imaging System (ROLIS) onboard Philae (Mottola et al. 2007, 2015) with those obtained by the Optical, Spectroscopic, and Infrared Remote Imaging System (OSIRIS, Keller et al. 2007) allowed the identification of the first touchdown point. In addition, Philae's Rosetta Lander Magnetometer and Plasma Monitor (ROMAP, Auster et al. 2007) provided precise timing of the various contact points through magnetic field measurements and subsequently identifying the final settling of the lander in Abydos site at 17:32 UT (Heinisch et al. 2015). During the nearly $57 \mathrm{~h}$ of prime Philae mission, the Comet Infrared and Visible Analyser camera (CIVA, Bibring et al. 2007) took images providing a panorama of the final landing site that revealed a rough terrain dominated by agglomerates of consolidated materials similar to $\mathrm{cm}$-sized pebbles (Bibring et al. 2015; Poulet et al. 2015a). After Philae exhausted its remaining energy on 15 November 2014, a huge multi-instrument attempt was performed to determine the exact lander location. Thanks to the Comet Nucleus Sounding Experiment by Radio wave Transmission experiment (CONSERT, Kofman et al. 2007), it was possible to indirectly identify the location of the lander within an ellipse of $16 \times 160 \mathrm{~m}$ in size by using the radio signals that are sent between Philae and Rosetta. This ellipse is located just outside the rim of the Hatmehit depression. Afterward, the comparison between OSIRIS Narrow Angle Camera (NAC) images taken before and after the landing allowed the possible identification of Philae on images acquired in mid-December 2014 at a distance of approximately $20 \mathrm{~km}$ (ESA Rosetta blog 2015). The aim of this work is to investigate the geomorphological properties and spectrophotometric modeling of the Abydos site on the OSIRIS images by providing a detailed description of the area that is assumed to surround Philae. This regional context study is useful to support Philae's measurements with the primary goal to supply the interpretation of the CIVA data analysis.

\section{Dataset}

The putative Philae position was reported as a few bright pixels on OSIRIS NAC images acquired in mid-December 2014, in 

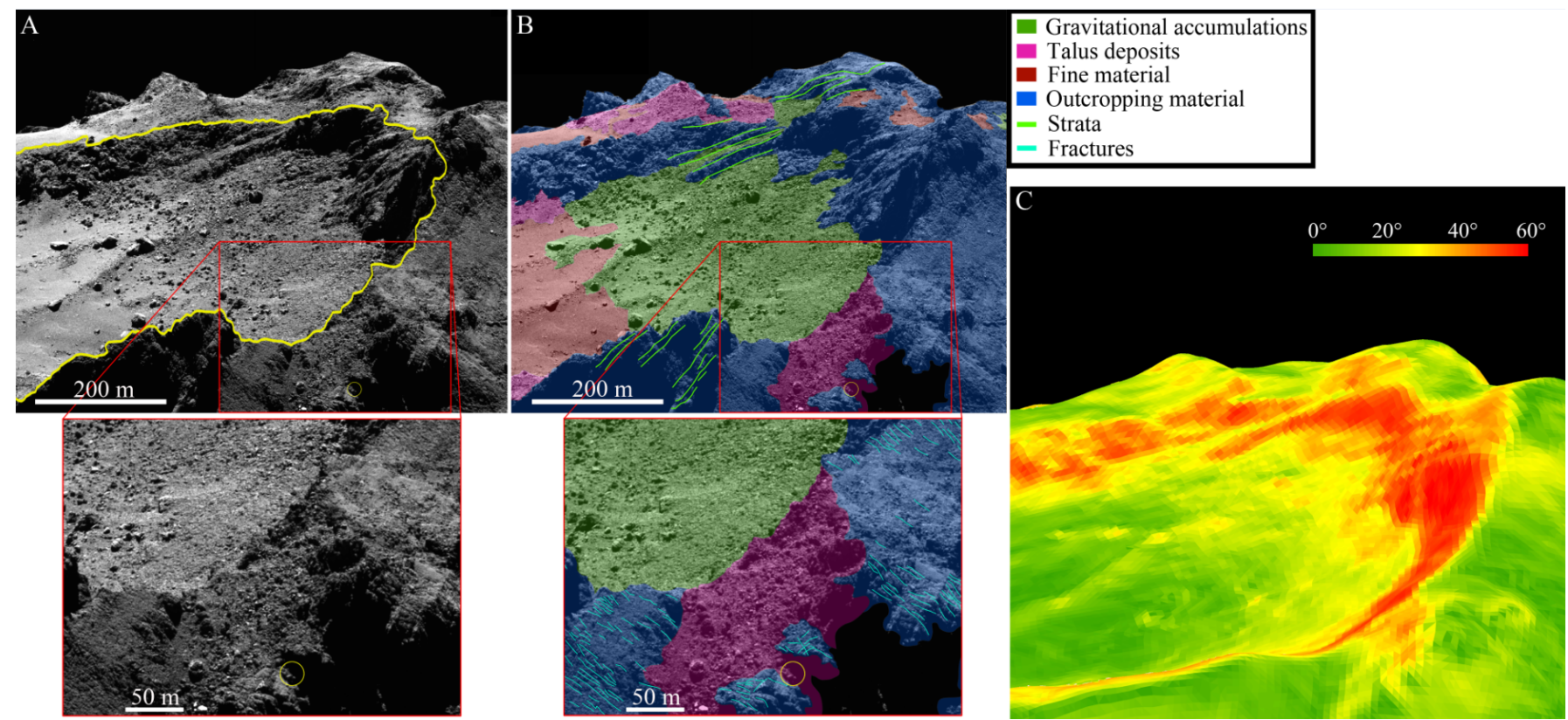

Fig. 1. (A) OSIRIS NAC image acquired on 6 December 2014 at 10:52:53 UT at an average phase angle of $90^{\circ}$ (top panel) with a close-up of the Abydos site (bottom). The region surrounding the putative landing site of Philae is identified here by a yellow circle, while the Hatmehit depression is outlined in yellow in the top panel. (B) The geomorphological map identifying the main geological units, produced on the image shown in the top panel of Fig. 1A, is displayed in the top panel with the close-up of the Abydos site showing both geomorphological units and linear features (bottom panel). (C) Gravitational slope map of the image obtained using the 3D shape model, derived with the stereo-photoclinometry (SPC) technique, and the gravitational potential model derived assuming a homogeneous nucleus and accounting for the centrifugal force that is due to the nucleus rotation.

very close proximity to the ellipse derived from the CONSERT instrument (ESA Rosetta blog 2015). Some bright pixels at the same location were also seen on another OSIRIS NAC image taken on 6 December 2014 at 10:52:53 UT (Fig. 1A, top panel). The image was acquired using the orange filter $(649.2 \mathrm{~nm})$ with a phase angle of about $90^{\circ}$ and at a distance of approximately $20 \mathrm{~km}$ above the nucleus surface, resulting in a spatial scale of $38.7 \mathrm{~cm} /$ pixel. Even if Philae is expected to be in the shadow part, we chose this image because its better viewing geometry allows a more accurate analysis of the area surrounding the lander. We describe the characteristics of the Abydos region including the geomorphological map, the gravitational slope map, and the size-frequency distribution of the boulders. The albedo and reddening maps were derived using the image acquired on 6 December 2014 at 10:53:04 UT in the blue filter $(480.7 \mathrm{~nm})$, in addition to the image acquired in the orange filter $(649.2 \mathrm{~nm})$.

\section{Geomorphological analysis}

We present in Fig. 1 the geomorphological map of the Abydos site and its close surroundings. The site where Philae came to rest is located on the small lobe of 67P, next to the Hatmehit depression (Thomas et al. 2015). We made use of the image's high spatial resolution to identify distinct geomorphological units.

1. On the Hatmehit depression, we confirm the results of La Forgia et al. (2015) for the distinction between the gravitational accumulation deposits (green unit, Fig. 1B) and the fine material covering the layered consolidated terrain (pink unit, Fig. 1B).

2. The surrounding structure of Hatmehit is characterized by outcropping material that is often layered as well as fractured (mapped as blue unit, Fig. 1B). The fractures are typically $30-50 \mathrm{~m}$ long, roughly parallel to each other, and show a preferred propagation orientation roughly perpendicular to the rim of Hatmehit. This fracturing pattern is similar to patterns observed in the neighboring Bastet region (Thomas et al. 2015; El-Maarry et al. 2015), but on a smaller scale. Fractures are ubiquitous on the surface of the comet, particularly in consolidated regions (El-Maarry et al. 2015). Therefore, their presence around the Abydos site is an indication of a high degree of consolidation in the region, whereas their propagation pattern suggests that they might be linked to the formation of the Hatmehit depression.

3. The Abydos landing region is a $0.02 \mathrm{~km}^{2}$ unconsolidated talus deposit distinct from the surrounding blue unit also by the presence of numerous boulders within what appears to be unconsolidated material. The suggested Philae landing site location is on this rocky deposit, but limited by two outcrops that limit its horizon (Bibring et al. 2015).

In addition, we analyzed the gravitational slopes of the Abydos site to describe the gravitational framework of the region using the 3D shape model of the comet, which was created on the basis of the entire NAC dataset that has provided multiple stereo coverage of the cometary surface. For our calculation we used the shape model derived with the stereo-photoclinometry (SPC) technique (Jorda et al. 2012). The gravitational slope is defined as the angle between the local surface normal pointing inside the nucleus and the gravity vector. The region surrounding the putative Philae position is found in correspondence with the high gravitational slope ranging from $20^{\circ}$ to $50^{\circ}$, which is commonly associated with collapse that is due to erosion by sublimation.

A large quantity of boulders is present in the Abydos region, specifically on the talus deposit. We identified the boulders with the same method as in Pajola et al. (2015b,a), who derived the first size-frequency distribution of boulders larger than $7 \mathrm{~m}$ on 67P. We manually identified these features as polygonal shapes by deriving their maximum length, that is, the diameter, and the 

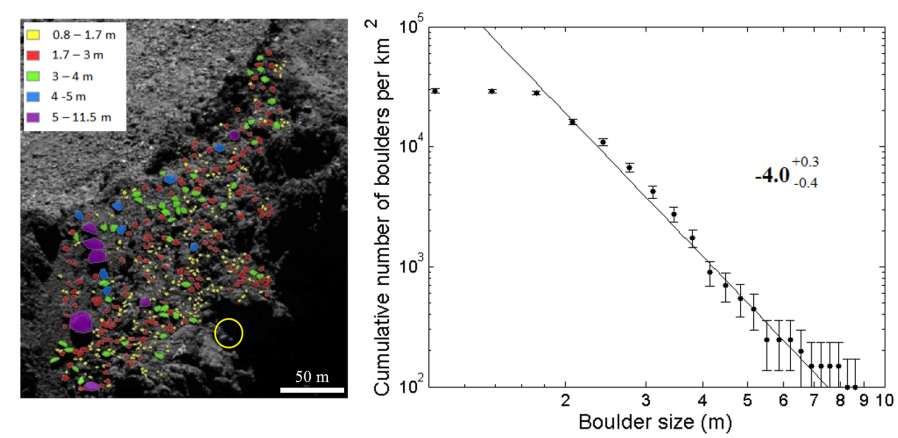

Fig. 2. Left panel: the spatial distribution of boulders on the Abydos site, where Philae is identified by a yellow circle. Boulders smaller than $1.7 \mathrm{~m}$, where the boulder size corresponds to the length calculated from the corresponding area, are shown in yellow and are not taken into account in the statistic. Right panel: the cumulative size-frequency distribution of boulders larger than $1.7 \mathrm{~m}$ identified on the Abydos site. The bin size is $34 \mathrm{~cm}$, and vertical error bars indicate the root of the cumulative number of counting boulders (as in Michikami et al. 2008). The fitting regression line gives a power-law index of $-4.0+0.3 /-0.4$.

corresponding area. By using the 3D SPC shape model, we geolocalized each boulder on the surface of the comet in terms of coordinates and distance (of about $\sim 18 \mathrm{~km}$ for each boulder); based on this, we calculated the exact diameter.

In this analysis, we considered boulders larger than $1.7 \mathrm{~m}$, which is a value above the three-pixel sampling that minimizes the likelihood of misidentifications (Nyquist 1928), even though we are also able to identify smaller features from their elongated shadow since the image was taken with a phase angle of $90^{\circ}$. We did not include boulders smaller than $1.7 \mathrm{~m}$ in the statistical analysis because the slope of the size-frequency distribution naturally decreases when it approaches the three-pixel detection limit (Mazrouei et al. 2014). The total number of boulders identified in this analysis is 447, 323 of which have diameters larger than $1.7 \mathrm{~m}$. Figure 2 shows the spatial distribution of boulders in the Abydos region. We obtained the cumulative size-frequency distribution per $\mathrm{km}^{2}$ using the corresponding area $\left(0.02 \mathrm{~km}^{2}\right)$ calculated from the 3D shape model of 67P (Preusker et al. 2015) through triangulating boulder point cloud. We then derived the cumulative size-frequency distribution of boulders using a constant-size bin of $34 \mathrm{~cm}$ (approximately one pixel resolution of the OSIRIS NAC image in the Abydos region at a distance of $18 \mathrm{~km}$ ). A power-law index of $-4.0+0.3 /-0.4$ is found (Fig. 2, right). The fitting regression line used to obtain the power-law index does not consider those points that are equally cumulatively repeated above $6 \mathrm{~m}$ because they represent a poor statistic that has not to be considered by the fit. This effect has previously been observed for larger boulder sizes as presented in Pajola et al. (2015b) and Michikami et al. (2008).

\section{Spectrophotometric modeling}

The reflectance of the entire region was computed as $\frac{I}{F}=$ $\pi r_{h}^{2} \frac{I_{\text {meas }}}{F_{\odot}}$, where $r_{h}$ is the heliocentric distance of the comet, $F_{\odot} / \pi$ is the solar irradiance at $1 \mathrm{AU}$, and $I_{\text {meas }}$ is the radiance of the comet measured in $\frac{W}{\mathrm{~m}^{2} \cdot \mathrm{nm} \cdot \mathrm{sr}}$ by OSIRIS images, calibrated following the standard pipeline described in Tubiana et al. (2015).

Since the images have been acquired at a phase angle of about $90^{\circ}$, the observed reflectance is highly influenced by the illumination and observing conditions. Therefore a photometric correction taking into account the topography and the

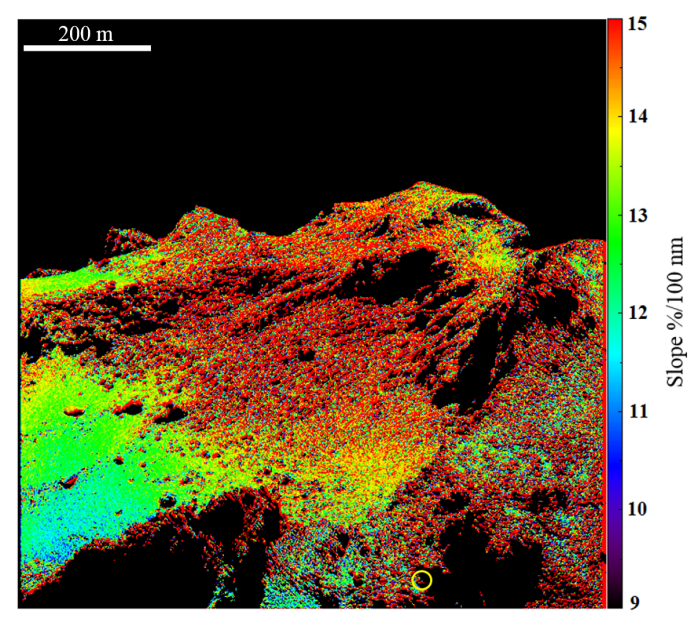

Fig. 3. Reddening map in percent/100 $\mathrm{nm}$ in the wavelength range of $480.7-649.2 \mathrm{~nm}$. The putative Philae position is identified here by a yellow circle.

illumination angles (see, for example, Fornasier et al. 2015; La Forgia et al. 2015) would be necessary to derive the normal albedo correctly. However, the particular geometry of the images did not allow this complex approach. We therefore decided to simply correct the observed reflectance by the effect of the average phase curve that was studied by Fornasier et al. (2015). Assuming that the averaged parameters of a Hapke model fitted by Fornasier et al. (2015) on the overall nucleus are also valid for the our region of interest, we applied the photometric correction using the model by Hapke $(1981,1984,2002)$.

We therefore calculated the normal albedo, $F_{\text {corr }}$, as

$\frac{I}{F_{\text {corr }}}=\frac{I}{F_{\text {meas }}} \cdot \frac{\frac{I}{F_{\text {Hapke }}}\left(\alpha=0^{\circ}\right)}{\frac{I}{F_{\text {Hapke }}}\left(\alpha=91^{\circ}\right)}$,

where $\frac{I}{F_{\text {Hapke }}}$ was computed using the disk-integrated Hapke expression and the parameters reported in Table 3 of Fornasier et al. (2015) for the orange and blue filter.

We combined the information by the two filters to compute the reddening, that is, the spectral slope in the wavelength range between the two filters. The orange and blue images were co-registered using a semi-automatic procedure available in the IDL-ENVI software, which linearly interpolates the input image to the reference image using a set of automatically generated control points based on a subset of user-defined tie points. After the images were aligned, we calculated the reddening in percent/100 $\mathrm{nm}$ in the wavelength range 480.7-649.2 $\mathrm{nm}$ using the following formulation (A'Hearn et al. 1984):

$S\left(\lambda_{b}, \lambda_{o}\right)=\frac{\frac{I}{F_{o}}-\frac{I}{F_{b}}}{\lambda_{o}-\lambda_{b}} \frac{20000}{\frac{I}{F_{b}}+\frac{I}{F_{o}}}$,

where $\frac{I}{F}$ is the reflectance of the surface (Hapke corrected) and the subscripts $b$ and $o$ refer to the blue and orange filters. The reddening map is shown in Fig. 3, and from the corresponding histogram distribution we obtain that the average reddening over the entire region is about $13.5 \% / 100 \mathrm{~nm}$, gradually changing to red from fine to rough material.

\section{Discussion and conclusion}

We provided a detailed analysis of the Abydos region, the proposed Philae resting site, to supply the interpretation of the 
Philae data analysis. To achieve this goal, we performed the geomorphological analysis of the region surrounding Philae in addition to spectrophotometrically modeling the area.

We identified different typologies of terrain. In particular, the putative Philae position is on a $0.02 \mathrm{~km}^{2}$ rocky deposit, limited by two regions of outcropping material that are fractured and layered. We recognized fractures in the Abydos region that suggest a brittle nature of the outcropping material and that often cut across strata heads. The evidence of these parallel linear features in the region agrees with the scenario that stratification is a dominant structural aspect of 67P throughout its entire bilobed shape (Massironi et al. 2015). As explained in Massironi et al. (2015), the comet stratification shows that the two lobes are the expression of two independent objects, perhaps formed as pebble-pile planetesimals, that are evidently characterized by onion-like stratification that is several hundred meters thick. Hence, the consolidated material strata belonging to outcrops can be representative of primordial stratification. This interpretation can support the hypothesis that Philae could be on primordial terrain. In this respect, the rough terrain dominated by agglomerates of consolidated materials similar to cm-sized pebbles (Poulet et al. 2015a), revealed by the CIVA image acquired by cameras 3 and 4 (Bibring et al. 2015), might be indicative of the formation process that formed the initial nucleus.

The resulting power-law index of the cumulative size-frequency distribution of the boulders is correlated with the morphological unit where the boulders are located. This unit is identified as the talus deposit, shown in Fig. 1, that has a gravitational source, and it could be interpreted as rockfall material deposit that originated from erosion, as supported by high slopes ranging from $20^{\circ}$ to $50^{\circ}$. We compared the powerlaw index obtained in this work $(-4.0+0.3 /-0.4)$ to the results of global and local boulders distribution of Pajola et al. (2015b), who classified the different size-frequency distribution on the basis of the possible acting formation processes. In addition, we pointed out that similar geomorphological settings on 67P comet show a similar power-slope distribution, suggesting that similar activity processes, pit formation or gravitational collapses and thermal fracturing events, most likely occurred in different areas of the comet and are responsible for the size-frequency distribution we see today (Pajola et al. 2015b). The studied talus deposit shows a similar size-frequency distribution as other talus deposits located in different areas of the comet, for instance, the one located at the boundary between Khepry and Ash and the deposit on Nut. Boulders of this type (called type II in Pajola et al. 2015b) are formed as a result of gravitational events triggered by sublimation and/or thermal fracturing that causes regressive erosion. Therefore, we suggest the same formation process for our case study as well, since the power law $(-4.0+0.3 /-0.4)$ is similar to the power-law index of the second type of boulders (power law from -3.5 to -4 ).

The size-frequency distribution may be compared with a similar quantitative analysis of pebble populations found on CIVA images. This comparison will be useful for understanding whether boulders and pebbles are produced by the same fragmentation process or if pebbles have a primordial origin, as suggested by the consolidated material strata, which might be representative of primordial stratification. The average value of the albedo, over the entire region, is $5.8 \%$ at $\lambda=480.7 \mathrm{~nm}$ and $7.4 \%$ at $\lambda=649.2 \mathrm{~nm}$, in agreement with the average albedo of the comet equal to $6.4 \%$ derived by Fornasier et al. (2015) and in particular with the albedo and the variations $(6.7 \pm 1)$ measured by La Forgia et al. (2015) in the analysis of Philae Agilkia landing site by OSIRIS data. The computed albedo is slightly higher than the one found by CIVA, which typically varies from 3 to $5 \%$ (Bibring et al. 2015). The average spectral slope across the area is $13.5 \% / 100 \mathrm{~nm}$, and the reddening map gradually changes to red from fine to rough material, which shows that outcrops and consolidated material are characterized by the reddest colors. This agrees with the average and local spectral slopes of the nucleus of 67P (Fornasier et al. 2015; La Forgia et al. 2015; Oklay et al. 2015).

The detailed investigation of the Abydos site presented in this work aids in the interpretation of the CIVA data (Poulet et al. 2015b) by providing the context of Philae landing site. The combination of information coming from the high-resolution analysis of OSIRIS images with those obtained by CIVA might offer insight into the pebble formation. This is fundamental for understanding whether physical processes, such as boulder formation or fragmentation activity, occur equally at different scales (m, $\mathrm{cm}$ or $\mathrm{mm}$ scale) on the comet, or if these agglomerates of consolidated material might have a primordial origin.

Acknowledgements. OSIRIS was built by a consortium of the Max-PlanckInstitut für Sonnensystemforschung, in Göttingen, Germany, CISAS-University of Padova, Italy, the Laboratoire d'Astrophysique de Marseille, France, the Instituto de Astrofísica de Andalucia, CSIC, Granada, Spain, the Research and Scientific Support Department of the European Space Agency, Noordwijk, The Netherlands, the Instituto Nacional de Técnica Aeroespacial, Madrid, Spain, the Universidad Politechnica de Madrid, Spain, the Department of Physics and Astronomy of Uppsala University, Sweden, and the Institut für Datentechnik und Kommunikationsnetze der Technischen Universität Braunschweig, Germany. The support of the national funding agencies of Germany (DLR), Italy (ASI), France (CNES), Spain (MEC), Sweden (SNSB), and the ESA Technical Directorate is gratefully acknowledged. We thank the ESA teams at ESAC, ESOC and ESTEC for their work in support of the Rosetta mission. We made use of the Arcgis 10.2 software together with the IDL and Matlab software to perform our analysis.

\section{References}

A'Hearn, M. F., Schleicher, D. G., \& Millis, et al. 1984, AJ, 89, 579 Auster, H. U., Apathy, I., Berghofer, G., et al. 2007, Space Sci. Rev., 128, 221 Bibring, J.-P., Lamy, P., Langevin, Y., et al. 2007, Space Sci. Rev., 128, 397 Bibring, J.-P., Langevin, Y., Carter, J., et al. 2015, Science, 349, 6247 Biele, J., Ulamec, S., Maibaum, M., et al. 2015, Science, 349, 6247 El-Maarry, M. R., Thomas, N., Gracia-Berná, A., et al. 2015, Geophys. Res. Lett., 42, 5170

ESA Rosetta blog 2015, The quest to find Philae

Fornasier, S., Hasselmann, P. H., Barucci, M. A., et al. 2015, A\&A, 583, A30

Hapke, B. 1981, J. Geophys. Res., 86, 3039

Hapke, B. 1984, Icarus, 59, 41

Hapke, B. 2002, Icarus, 157, 523

Heinisch, P., Auster, H.-U., \& Richter, I., et al. 2015, in EGU Abstracts, 12166 Jorda, L., Lamy, P. L., Gaskell, R. W., et al. 2012, Icarus, 221, 1089 Keller, H. U., Barbieri, C., Lamy, P., et al. 2007, Space Sci. Rev., 128, 433 Kofman, W., Herique, A., Goutail, J.-P., et al. 2007, Space Sci. Rev., 128, 413 La Forgia, F., Giacomini, L., Lazzarin, M., et al. 2015, A\&A, 583, A41 Massironi, M., Simioni, E., Marzari, F., et al. 2015, Nature, 526, 402 Mazrouei, S., Daly, M. G., \& Barnouin, et al. 2014, Icarus, 229, 181 Michikami, T., Nakamura, A. M., Hirata, N., et al. 2008, Earth Planets Space, 60, 13

Mottola, S., Arnold, G., Grothues, H.-G., et al. 2007, Space Sci. Rev., 128, 241 Mottola, S., Arnold, G., Grothues, H.-G., et al. 2015, Science, 349, 6247 Nyquist, H. 1928, Trans. Am. Inst. Electr. Eng., 47, 617 Oklay, N., Vincent, J. B., \& Fornasier, S., et al. 2015, A\&A, submitted Pajola, M., Lucchetti, A., \& Bertini, I., et al. 2015a, A\&A, in press DOI: $10.1051 / 0004-6361 / 201526834$

Pajola, M., Vincent, J.-B., Güttler, C., et al. 2015b, A\&A, 583, A37 Poulet, F., Bibring, J.-P., Carter, J., et al. 2015a, in EGU Abstracts, 9517 Poulet, F., Lucchetti, A., \& Bibring, J.-P., et al. 2015b, AAS/DPS Abstracts Preusker, F., Scholten, F., Matz, K.-D., et al. 2015, A\&A, 583, A33 Thomas, N., Sierks, H., Barbieri, C., et al. 2015, Science, 347

Tubiana, C., Güttler, C., Kovacs, G., et al. 2015, A\&A, 583, A46 
1 Center of Studies and Activities for Space, CISAS, "G. Colombo", University of Padova, via Venezia 15, 35131 Padova, Italy

e-mail: alice.lucchetti@oapd.inaf.it

2 INAF Osservatorio Astronomico di Padova, vic. dell'Osservatorio 5, 35122 Padova, Italy

3 Laboratoire d'Astrophysique de Marseille, UMR 7236, CNRS \& Aix-Marseille Université, 38 rue Frédéric Joliot-Curie, 13388 Marseille Cedex 13, France

${ }^{4}$ Institut d'Astrophysique Spatiale, CNRS/Univ. Paris Sud, 91440 Orsay Cedex, France

5 Department of Physics and Astronomy "G. Galilei", University of Padova, Vic. Osservatorio 3, 35122 Padova, Italy

6 Geosciences Department, University of Padova, via G. Gradenigo 6, 35131 Padova, Italy

7 Physikalisches Institut der Universität Bern, Sidlerstr. 5, 3012 Bern, Switzerland

8 Max-Planck-Institut fuür Sonnensystemforschung, Justus-vonLiebig-Weg 3, 37077 Göttingen, Germany

9 Centro de Astrobiologia, CSIC-INTA, 28850 Torrejon de Ardoz, Madrid, Spain

10 International Space Science Institute, Hallerstraße 6, 3012 Bern, Switzerland

11 Scientific Support Office, European Space Research and Technology Centre/ESA, Keplerlaan 1, Postbus 299, 2201 AZ Noordwijk ZH, The Netherlands

12 Department of Physics and Astronomy, Uppsala University, Box 516, 75120 Uppsala, Sweden
13 PAS Space Research Center, Bartycka 18A, 00716 Warszawa, Poland

14 Institut fur Geophysik und extraterrestrische Physik, TU Braunschweig, 38106 Braunschweig, German

15 University of Maryland, Department of Astronomy, College Park, MD 20742-2421, USA

16 LESIA-Observatoire de Paris, CNRS, UPMC, Univ. Paris 06, Univ. Paris-Diderot, 5 Place J. Janssen, 92195 Meudon Principal Cedex, France

17 LATMOS, CNRS/UVSQ/IPSL, 11 Boulevard d'Alembert, 78280 Guyancourt, France

18 CNR-IFN UOS Padova LUXOR, via Trasea 7, 35131 Padova, Italy

19 Department of Industrial Engineering, University of Padova, via Venezia 1, 35131 Padova, Italy

${ }^{20}$ University of Trento, via Sommarive 9, 38123 Trento, Italy

21 INAF Osservatorio Astronomico di Trieste, via Tiepolo 11, 34014 Trieste, Italy

22 Instituto de Astrofísica de Andalucía-CSIC, 18008 Granada, Spain

23 Deutsches Zentrum für Luft- und Raumfahrt (DLR), Institut für Planetenforschung, Rutherfordstraße 2, 12489 Berlin, Germany

24 National Central University, Graduate Institute of Astronomy, 300 Chung-Da Rd, 32054 Chung-Li, Taiwan

25 Operations Department, European Space Astronomy Centre/ESA, PO Box 78, 28691 Villanueva de la Canada, Madrid, Spain

26 Department of Information Engineering, University of Padova, via Gradenigo 6/B, 35131 Padova, Italy 